\title{
Probing non-standard neutrino-electron interactions with solar and reactor neutrinos
}

\author{
A. Bolaños ${ }^{1}$, O. G. Miranda ${ }^{1}$, A. Palazzo ${ }^{2}$, M. A. Tórtola ${ }^{3}$, and J. W. F. Valle ${ }^{2}$ \\ 1 Departamento de Física, Centro de Investigación y de Estudios Avanzados del IPN, \\ Apt. Postal 14-740 07000 México, D F, México \\ ${ }^{2}$ AHEP Group, Institut de Física Corpuscular - C.S.I.C./Universitat de València \\ Edificio Institutos de Paterna, Apt. 22085, E-46071 Valencia, Spain \\ ${ }^{3}$ II. Institut für theoretische Physik, Universität Hamburg, \\ Luruper Chausee 149, 22761 Hamburg, Germany
}

\begin{abstract}
Most neutrino mass extensions of the standard electroweak model entail non-standard interactions which, in the low energy limit, can be parametrized in term of effective four-fermion operators $\nu_{\alpha} \nu_{\beta} \bar{f} f$. Typically of sub-weak strength, $\epsilon_{\alpha \beta} G_{F}$, these are characterized by dimensionless coupling parameters, $\epsilon_{\alpha \beta}$, which may be relatively sizeable in a wide class of schemes. Here we focus on non-universal (NU) flavor conserving couplings $(\alpha=\beta)$ with electrons $(f=e)$ and analyse their impact on the phenomenology of solar neutrinos. We consistently take into account their effect both at the level of propagation where they modify the standard MSW behavior, and at the level of detection, where they affect the cross section of neutrino elastic scattering on electrons. We find limits which are comparable to other existing model-independent constraints.
\end{abstract}

PACS numbers: 14.60.Pq, 13.15.+g 


\section{INTRODUCTION}

Solar neutrino oscillations dominated by matter effects [1, 2] are currently well established by solar neutrino experiments [3, 4, 5, 6, 7, 8, 9, 10, 11, 12, 13, 14, 15, 16, 17, 18, 19, 20, 21, 22] and have been confirmed by the long-baseline KamLAND reactor experiment [23, 24, 25]. The combination between solar and KamLAND determines a unique solution in the massmixing parameter space, the so-called Large Mixing Angle (LMA) solution, see e.g. |26, 27, 28, 29]. This solution has been shown to be quite robust against possible uncertainties in solar physics, such as magnetic fields in the radiative zone, that could give rise to noise fluctuations [30, 31, 32, 33, 34, 35, 36, 37], as well as in the convective zone [38, 39], that could induce spin-flavor neutrino conversions [40, 41]. The KamLAND data play a crucial role in establishing that non-standard effects can play only a subleading role [42], their amplitude being effectively constrained.

Altogether, the high precision and robustness of the current data render solar and reactor neutrinos a unique probe of possible physics beyond the Standard Model [42, 43, 44, 45, 46, 47, 48], complementing information from atmospheric and accelerator neutrinos [49, 50]. Moreover non-standard interactions provide an important window of opportunity for current or upcoming long-baseline neutrino oscillation experiments, and have been extensively considered in this framework [51, 52, 53, 54, 55, 56, 57].

It is worth stressing that, while constrained by the solar and KamLAND data, nonstandard interactions (NSI) provide an exception to robustness of the neutrino oscillation interpretation [45, 46] and they might even shift the solution to the so-called dark side region of the neutrino parameter space [58]. Indeed, with oscillations still being the underlying mechanism, an additional degenerate oscillation solution in neutrino oscillation parameters can appear for sufficiently intense non-standard interactions

Neutrino NSI constitute an unavoidable feature of gauge models of neutrino mass, for ex-

ample models of the generic seesaw type [59] where neutrino masses arise from the admixture of isodoublet and isosinglet neutral leptons. In general, the lepton mixing matrix for charged currents is described by a matrix, $K$, and the corresponding neutral weak interactions are described by a non-trivial matrix [59] $K^{\dagger} K$. In particular, in the simplest type-I seesaw schemes [60, 61, 62, 63], the smallness of neutrino mass implies that, barring fine-tuning, the magnitude of neutrino NSI and its effects are expected to be negligible. However this 
need not be always the case. For example, by a suitable symmetry one may prevent the appearance of type-I seesaw mass contributions, hence allowing for the new neutral heavy leptons to lie at a mass scale accessible to accelerator experiments and, simultaneously, potentially produce sizeable NSI strengths. For example, this may happen in some specially designed triplet (type-II) seesaw models [59, 64], as shown in Ref. [65].

Alternatively, one may extend the lepton sector of the $S U(2) \otimes U(1)$ theory by adding a set of two 2-component isosinglet neutral fermions in each generation [66, 67]. This scheme is sometimes called "inverse seesaw" an provides an elegant way to generate small neutrino masses without a super-heavy scale. This automatically allows for a sizeable magnitude of neutrino NSI strengths, unconstrained by the smallness of neutrino masses ${ }^{1}$. The NSI which are engendered in this case will necessarily affect neutrino propagation properties in matter, an effect that may be resonant in certain cases [71, 72, 73]. They may also be large enough as to produce effects in the laboratory.

Another possible way to induce neutrino NSI is in the context of low-energy supersymmetry without R-parity conservation [74, 75, 76, 77] both of the bilinear [78, 79, 80, 81] and trilinear type [82]. The smallness of neutrino masses may also follow from its radiative nature [83, 84], allowing for possibly sizeable NSI strengths ${ }^{2}$.

In general one may consider a general class of non-standard interactions described via the effective four fermion Lagrangian,

$$
-\mathcal{L}_{\mathrm{NSI}}^{e f f}=\varepsilon_{\alpha \beta}^{f P} 2 \sqrt{2} G_{F}\left(\bar{\nu}_{\alpha} \gamma_{\rho} L \nu_{\beta}\right)\left(\bar{f} \gamma^{\rho} P f\right)
$$

where $G_{F}$ is the Fermi constant and $\varepsilon_{\alpha \beta}^{f P}$ parametrize the strength of the NSI. The chiral projectors $P$ denote $\left\{R, L=\left(1 \pm \gamma^{5}\right) / 2\right\}$, while $\alpha$ and $\beta$ denote the three neutrino flavors, $e, \mu$ and $\tau$ and $f$ is a first generation $\operatorname{SM}$ fermion $(e, u$ or $d)$.

For example, the existence of effective neutral current interactions contributing to the neutrino scattering off $d$-quarks in matter, provides new flavor-conserving as well as flavorchanging terms for the matter potentials of neutrinos. Such NSI are directly relevant for solar [46, 58, 87] and atmospheric neutrino propagation [49, 50, 88].

In general, the presence of NSI affects the solar neutrino phenomenology inducing pro-

\footnotetext{
${ }^{1}$ It also provides an explicit example for flavour and $\mathrm{CP}$ violation completely detached from the smallness of neutrino masses [68, 69, 70].

${ }^{2}$ For an alternative recent discussion of possible NSI strengths in a similar context see Refs. [85, 86]
} 
found modifications both in matter propagation [71, 89, 90] as well as in the detection process [43]. Although various works have investigated the effects of NSI at the level of propagation inside the Sun [45, 46, 58], the impact of NSI at the level of detection has received far less attention and only qualitative studies have been performed so far [43, 44] ${ }^{3}$.

Therefore, it seems timely and interesting to investigate in more detail NSI trying to fill this gap in the literature. Our main aim is then to perform a quantitative analysis of the impact of NSI in solar neutrino phenomenology consistently taking into account their impact both on propagation and on detection processes. The simultaneous inclusion of NSI effects in both processes unavoidably renders the computational analysis very demanding since for each choice of the NSI couplings, one has to convolve the oscillation probability with crosssection of the relevant process. For definiteness in this work we have restricted our study to the following situation: I) We have considered only non universal (NU) flavor conserving interactions neglecting flavor changing neutral current interactions (FCNC). II) We have considered interactions only with electrons $(f=e)$. III) We have performed our analysis switching on the interaction for one neutrino flavor at a time. IV) We do not consider NSI of $\nu_{\mu}$ with electrons since the current bounds in this case [48] $\left(-0.033 \leq \varepsilon_{\mu \mu}^{L} \leq 0.055\right.$, $-0.040 \leq \varepsilon_{\mu \mu}^{R} \leq 0.053$ ) are stronger than the attainable sensitivity from our solar analysis.

A final remark is in order. In general, one should also consider the possible simultaneous presence of FCNC, and include NSI with up and/or down quarks ${ }^{4}$. We have not performed such a general analysis since the number of parameters would disproportionally increase. Although considering only flavor preserving NSI with electrons may seem somewhat reductive, we deem that a model-indpendent detailed study of this specific case may provide particular insight and may be useful for future, more complete, studies.

The paper is organized as follows. In Sec. II we discuss the impact of NU non-standard interactions on propagation properties providing quantitative constraints on their amplitude. In Sec. III we consider the effect of NSI on the elastic scattering cross section. In section IV we discuss the general case in which we simultaneously include NSI both in the propagation and in detection of electron neutrinos. In Sec. $\mathrm{V}$ we show analogous results for the case of $\tau$ neutrinos. Finally, in Sec. VI we trace our conclusions.

\footnotetext{
${ }^{3}$ Solar and reactor neutrino fluxes are unaffected by the class of NSI which typically arise in models of neutrino mass.

${ }^{4}$ Limits on NSI involving up and down quarks have already been reported in the literature [49, 50, 58$]$.
} 


\section{NON-STANDARD PROPAGATION}

In this section we introduce the basic formalism describing neutrino propagation in the presence of non-standard interactions and derive quantitative bounds on the amplitude of the effective non-universal couplings. These bounds will be an important ingredient to interpret the results of our full analysis presented in Sec. IV and Sec. V where we consider the interplay of NSI effects in propagation and detection processes.

Here and in the following, we assume the standard parametrization for the lepton mixing matrix [59], within the convention adopted by the Particle Data Group [91], setting the small mixing angle $\theta_{13}$ to zero for the sake of simplicity. For $\theta_{13}=0$, standard oscillations in the $\nu_{e} \rightarrow \nu_{e}$ channel probed by long-baseline reactor (KamLAND) and by solar neutrino experiments are driven by only two parameters: the mixing angle $\theta_{12}$ and the neutrino squared mass difference $\Delta m_{21}^{2}=m_{2}^{2}-m_{1}^{2}$. In the flavor basis, the evolution of neutrinos can be written as

$$
i \frac{d}{d x}\left(\begin{array}{c}
\nu_{e} \\
\nu_{a}
\end{array}\right)=H\left(\begin{array}{c}
\nu_{e} \\
\nu_{a}
\end{array}\right),
$$

where $\nu_{a}$ is a linear superposition of $\nu_{\mu}$ or $\nu_{\tau}$, and $H$ is the total hamiltonian

$$
H=H_{\mathrm{kin}}+H_{\mathrm{dyn}}^{\mathrm{MSW}}+H_{\mathrm{dyn}}^{\mathrm{NSI}}
$$

split as the sum of the kinetic term, the standard Mikheev-Smirnov-Wolfenstein (MSW) matter term [1, 2] and of a new, NSI-induced, matter term [71]. The kinetic term depends on the mixing angle $\theta_{12}$, on the squared mass difference $\Delta m_{21}^{2}=m_{2}^{2}-m_{1}^{2}$, and on the energy $E$ as

$$
H_{\text {kin }}=\frac{k}{2}\left(\begin{array}{cc}
-\cos 2 \theta_{12} & \sin 2 \theta_{12} \\
\sin 2 \theta_{12} & \cos 2 \theta_{12}
\end{array}\right)
$$

where $k=\Delta m_{21}^{2} / 2 E$ is the neutrino oscillation wave number. The standard (MSW) interaction term can be expressed as

$$
H_{\mathrm{dyn}}^{\mathrm{MSW}}=V(x)\left(\begin{array}{ll}
1 & 0 \\
0 & 0
\end{array}\right)
$$

where $V(x)=\sqrt{2} G_{F} N_{e}(x)$ is the effective potential induced by interaction with the electrons with number density $N_{e}(x)$. The NSI term can be cast in the form

$$
H_{\mathrm{dyn}}^{\mathrm{NSI}}=V(x)\left(\begin{array}{cc}
0 & \varepsilon \\
\varepsilon & \varepsilon^{\prime}
\end{array}\right)
$$


where $\varepsilon$ and $\varepsilon^{\prime}$ are two effective parameters that, neglecting $\varepsilon_{\alpha \mu}^{f P}$, are related with the vectorial couplings by:

$$
\varepsilon=-\sin \theta_{23} \varepsilon_{e \tau}^{e V} \quad \varepsilon^{\prime}=\sin ^{2} \theta_{23} \varepsilon_{\tau \tau}^{e V}-\varepsilon_{e e}^{e V}
$$

In the present work we focus on the flavor conserving non-universal (NU) couplings, setting the flavor-changing off-diagonal coupling $\varepsilon=0$. Hence, in the treatment of solar neutrino propagation, in addition to the mass-mixing parameters we include the coupling $\varepsilon^{\prime}$.

In our numerical analysis we have included the data from the radiochemical experiments Homestake [3], Sage [5] and GALLEX/GNO [6, 7, 8], from Super-KamioKande (SuperK) [10, 11, 12], from all three phases of the Sudbury Neutrino Observatory (SNO) [14, 15, 16, 17, 18, 19], and from Borexino [21]. We have also included the latest KamLAND data [25] using a threshold of $2.6 \mathrm{MeV}$, which allows us to neglect the contribution of lowenergy geo-neutrinos.

It is worth noticing that although we have incorporated both standard and non-standard matter effects, due to the low matter density of the Earth crust, they have only a negligible effect in KamLAND, for the range of parameters we are considering. Therefore the inclusion of KamLAND in the analysis has the important effect of determining the solar mass-mixing parameter, independently of the non-standard interaction parameters.

In Fig. 1 we show the constraints we obtain on the parameter $\varepsilon^{\prime}$ from the solar neutrino data in combination with KamLAND after marginalization over the two mass-mixing parameters. We can qualitatively explain these bounds as follows. We notice that, since the term containing the effective NU coupling is diagonal, it is formally equivalent to a redefinition of the potential $\mathrm{V}^{5}$,

$$
V(x) \rightarrow\left(1-\varepsilon^{\prime}\right) V(x)
$$

In the LMA region the propagation is adiabatic so that, up to small Earth matter effects, the $\nu_{e}$ survival probability is given by the simple formula

$$
P_{e e}=\frac{1}{2}\left(1+\cos 2 \tilde{\theta}_{12}\left(x_{0}\right) \cos 2 \theta_{12}\right),
$$

\footnotetext{
${ }^{5}$ As shown in [92], the uncertainty in the solar composition leads to a small uncertainty on the electron neutrino density (and then on the potential V). In the region relevant for adiabatic transitions of solar neutrinos $R<0.6$ (in units of solar radii) this can be quantified as less than $2 \%$, hence negligible in the context of our analysis.
} 


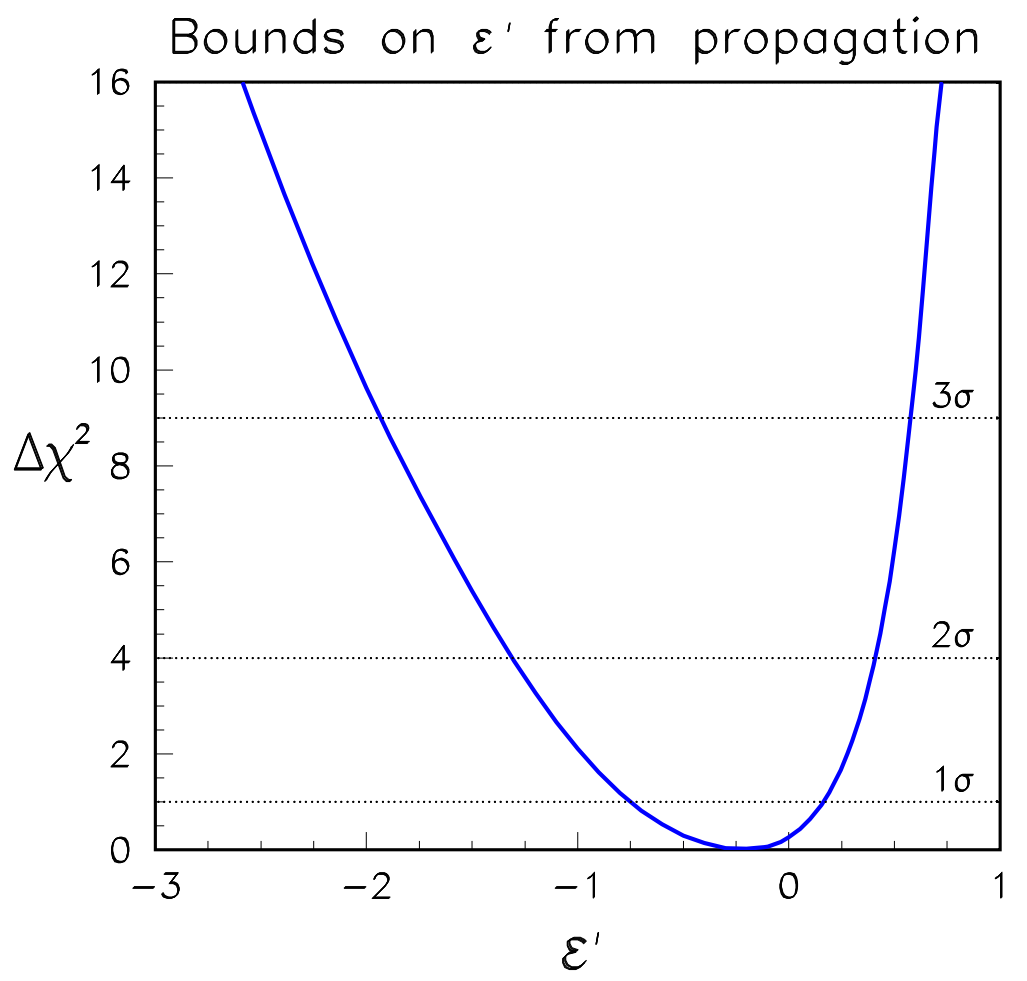

FIG. 1: Constraints on the effective amplitude characterizing NU non-standard interactions in propagation.

where $\tilde{\theta}_{12}\left(x_{0}\right)$ is the energy-dependent effective mixing angle in matter at the production point $x_{0}$ (see, e.g., [93] and references therein),

$$
\cos 2 \tilde{\theta}_{12}\left(x_{0}\right)=\frac{\cos 2 \theta_{12}-V\left(x_{0}\right) / k}{\sqrt{\left(\cos 2 \theta_{12}-V\left(x_{0}\right) / k\right)^{2}+\sin ^{2} 2 \theta_{12}}} .
$$

From the equations above we see that the survival probability depends on the potential $V(x)$ through the ratio $V / k$, and a rescaling of $V$ can be compensated by a rescaling of the wave number $k$, which for a fixed neutrino energy implies a rescaling of the value of $\Delta m_{21}^{2}$ preferred by data. Therefore, in the presence of a small NU coupling the LMA solution moves upward $\left(\varepsilon^{\prime}<0\right)$ or downward $\left(\varepsilon^{\prime}>0\right)$ in the mass-mixing parameter space (not shown). Now we note that in the absence of non-standard interactions the value of $\Delta m_{21}^{2}$ preferred by solar data is in agreement to the one identified with high precision by KamLAND. Hence, the presence of the additional non-standard effects tends to spoil this agreement and the tension arising between solar and KamLAND effectively constrains the 
amplitude of $\varepsilon^{\prime} 6$. It is interesting to note that the constraints on such parameter have now reached the "sensitivity limit" attainable by KamLAND high precision measurements [25]. Indeed, we have checked that the constraints that one would obtain fixing the $\Delta m_{21}^{2}$ at the best fit obtained by KamLAND are practically equivalent to those we obtain by exact marginalization. The freedom for $\varepsilon^{\prime}$ is essentially determined by the range of $\Delta m_{21}^{2}$ allowed by the solar data alone. Indeed, by varying the value of $\varepsilon^{\prime}$, the wide solar LMA solution smoothly "slides" over the thin $\Delta m_{21}^{2}$ region determined by KamLAND.

We observe that while for small deviations around the standard value $\left(\varepsilon^{\prime}=0\right)$ the bounds are symmetrical, for larger amplitudes the constraints becomes asymmetrical, i. e. stronger for positive values of $\varepsilon^{\prime}$. This behavior is due to the typical shape of the solar LMA solution (see for example [26, 28]) which is more (less) elongated towards large (low) $\Delta m_{21}^{2}$ values. Indeed, the solar LMA solution is strongly limited from below by the (non) observation of day-night asymmetry in Super-K and SNO and it is constrained in the upper part essentially by the $\mathrm{CC} / \mathrm{NC}$ ratio measured by SNO. This asymmetric behavior will be relevant when considering (see Sec. IV and V) the interplay among the limits coming from non-standard propagation with those coming from non-standard detection.

\section{NON-STANDARD DETECTION}

Non-standard couplings of neutrinos with electrons affect the elastic scattering $\left(\nu_{a} e \rightarrow\right.$ $\left.\nu_{a} e\right)$ process modifying the number of events and their spectral distribution expected in the Super-K detector and to a much lesser extent in the SNO detector. In principle they also affect the Borexino spectrum but we have checked that the current statistics is (still) too low to compete with Super-K.

The standard differential cross section for $\left(\nu_{a} e \rightarrow \nu_{a} e\right)$ scattering processes has the well known form

$$
\frac{d \sigma_{a}^{\mathrm{std}}}{d T}\left(E_{\nu}, T_{e}\right)=\frac{2 G_{F}^{2} m_{e}}{\pi}\left[\left(g_{1}^{a}\right)^{2}+\left(g_{2}^{a}\right)^{2}\left(1-\frac{T_{e}}{E_{\nu}}\right)^{2}-g_{1}^{a} g_{2}^{a} \frac{m_{e} T_{e}}{E_{\nu}^{2}}\right],
$$

where $m_{e}$ is the electron mass, $E_{\nu}$ is the incident neutrino energy, $T_{e}$ is the electron recoil energy. The quantities $g_{1}^{a}$ and $g_{2}^{a}$ are related to the SM neutral current couplings of the

\footnotetext{
${ }^{6}$ This behavior was indeed already noticed in Ref. [94], where upper bounds on possible deviations from the standard amplitude of the MSW interaction potential were considered.
} 
electron $g_{L}^{e}=-1 / 2+\sin ^{2} \theta_{W}$ and $g_{R}^{e}=\sin ^{2} \theta_{W}$, with $\sin ^{2} \theta_{W}=0.23119[91]^{7}$. For $\nu_{\mu, \tau}$ neutrinos, which take part only in neutral current interactions we have $g_{1}^{\mu, \tau}=g_{L}^{e}, g_{2}^{\mu, \tau}=g_{R}^{e}$, while for electron neutrinos both charge current (CC) and neutral current (NC) interactions are present and $g_{1}^{e}=1+g_{L}^{e}, g_{2}^{e}=g_{R}^{e}$. In the presence of NU non-standard interactions the cross section can be written in the same form of Eq. (11) but with $g_{1,2}^{a}$ replaced by the effective non-standard couplings $\tilde{g}_{1}^{a}=g_{1}^{a}+\varepsilon_{a a}^{e L}$ and $\tilde{g}_{2}^{a}=g_{2}^{a}+\varepsilon_{a \alpha}^{e R}$.

Strong limits can be placed on $\nu_{\mu}$ interactions with electrons [48] $\left(-0.033 \leq \varepsilon_{\mu \mu}^{L} \leq 0.055\right.$, $\left.-0.040 \leq \varepsilon_{\mu \mu}^{R} \leq 0.053\right)$. In contrast, the constraints on the other two NU couplings are rather loose [48]. Therefore in our analysis we can safely neglect NSI with muons of either helicity, and focus in what follows on possible non-standard couplings of $\nu_{e}$ and $\nu_{\tau}$. In addition we have performed our analysis switching on one flavor non-standard interaction at a time, due to computational limits. Indeed, already in this simple case we must consider as additional parameters $\varepsilon_{a a}^{e L}$ as well as $\varepsilon_{a a}^{e R}$ at the level of detection, and their sum at the level of propagation.

Before introducing our numerical results it is worth discussing the qualitative behavior one expects when NU interactions are present in the detection process. We first observe that for the high energy Boron neutrinos (which are relevant for Super-K) MSW matter effects dominate and the survival probability is approximately $P_{e e} \sim \sin ^{2} \theta_{12} \sim 1 / 3$. Furthermore, the transition probabilities to the other flavors are approximately equal $\left(P_{e \mu} \sim P_{e \tau} \sim 1 / 3\right)$ since the admixture of $\nu_{\mu}$ and $\nu_{\tau}$ neutrinos is determined by the nearly maximal "atmospheric" mixing angle [26, 27, 28, 29] $\left(\sin ^{2} \theta_{23} \sim 0.5\right)$. Hence, up to small earth matter effects, an approximately equal admixture of the three neutrino flavors arrives at the SuperKamiokande detector. Therefore from Eq. (11) one can expect the following general features: I) In both cases of $\nu_{e}$ and $\nu_{\tau}$ interactions, a deviation of the L-type coupling should mostly affect the total rate through the first term in Eq. (11). II) The relative contribution of the first term in the cross section is almost one order of magnitude larger for $\nu_{e}$ compared to $\nu_{\tau}$ $\left(\left(g_{1}^{e}\right)^{2} /\left(g_{1}^{\tau}\right)^{2} \simeq 7\right)$. Thus we expect this feature to be reflected in a reduced sensitivity to $\varepsilon_{\tau \tau}^{e L}$ compared to $\varepsilon_{e e}^{e L}$. III) Deviations of the R-type coupling will instead modify the expected energy spectrum through the second term and (to a lesser extent) through the third term.

\footnotetext{
${ }^{7}$ For our numerical analysis, instead of this simple tree level expression, we also include the radiative corrections given in Ref. [95].
} 
IV) The value of $g_{2}^{a}$ is identical for $\nu_{e}$ and $\nu_{\tau}$ and we expect comparable sensitivities for the $\varepsilon_{e e}^{e R}, \varepsilon_{\tau \tau}^{e R}$ effective couplings coming from the Super-K spectral information. V) The third term (proportional to $g_{1}^{a} g_{2}^{a}$ ) is suppressed by the (energy dependent) factor $m_{e} T_{e} / E_{\nu}^{2}$, and should induce non negligible effects only in the case of electron neutrinos $(a=e)$ since in this case $g_{1}^{a}$ is bigger $\left(g_{1}^{e} \sim 0.73\right.$ in the standard case).

\section{CONSTRAINTS ON ELECTRON NEUTRINO INTERACTIONS}

In this section we present the numerical results of our analysis in the presence of $\mathrm{NU}$ couplings of $\nu_{e}$ with electrons. With this aim we have performed a joint analysis of solar and KamLAND data in the $\left(\Delta m_{21}^{2}, \sin ^{2} \theta_{12}, \varepsilon_{e e}^{e L}, \varepsilon_{e e}^{e R}\right)$ parameter space, taking into account that only the vectorial combination $\varepsilon_{e e}^{e V}=\varepsilon_{e e}^{e L}+\varepsilon_{e e}^{e R}$ is involved in the propagation. Moreover, we have limited our scan in the L-type NSI parameter, $\varepsilon_{e e}^{e L}$, to the range $(-0.3,0.3)$. Although a degeneracy in the value of this parameter appears when one includes only the $\nu e$ scattering data [47], allowing for NSI values as large as $\varepsilon_{e e}^{e L}=-1.5$, these values turn out to be forbidden when one also includes the LEP data, as shown in Ref. [48].

In the three panels of Fig. 2 2 we show the regions allowed in the plane $\left[\varepsilon_{e e}^{e L}, \varepsilon_{e e}^{e R}\right]$ where the mass-mixing parameters have been marginalized away. In the left panel we show the region allowed when we switch on the non-standard effects only in the detection process. The sensitivity to deviations of the L-type coupling is higher than the R-type sensitivity (notice the different scale used for the two parameters). This behavior follows from the fact that the most important effect of $\varepsilon_{e e}^{e L}$ arises from the first term in Eq. (11) and approximately consists in an energy independent rescaling of the cross section. This in turn leads to deviations of the predicted theoretical values of the total Super-K rate which are rejected by all the remaining solar data. To better understand this point we note that, if only Super-K data were included in the analysis, large deviations of the total cross section could be allowed since they could be compensated by a rescaling of the theoretical boron flux which is still uncertain at the $\sim 20 \%$ level. However, the combination of the Super-K data with the other solar neutrino experiments drastically improves the sensitivity to $\varepsilon_{e e}^{e L}$. In particular SNO plays a crucial role in this respect, limiting possible departures of the total Super-K rate in two ways. First, the $\mathrm{NC}$ measurement provides a direct measurement of the boron flux in agreement with the SSM prediction to within $\sim 6 \%$ or so, effectively reducing the allowed space for a possible 

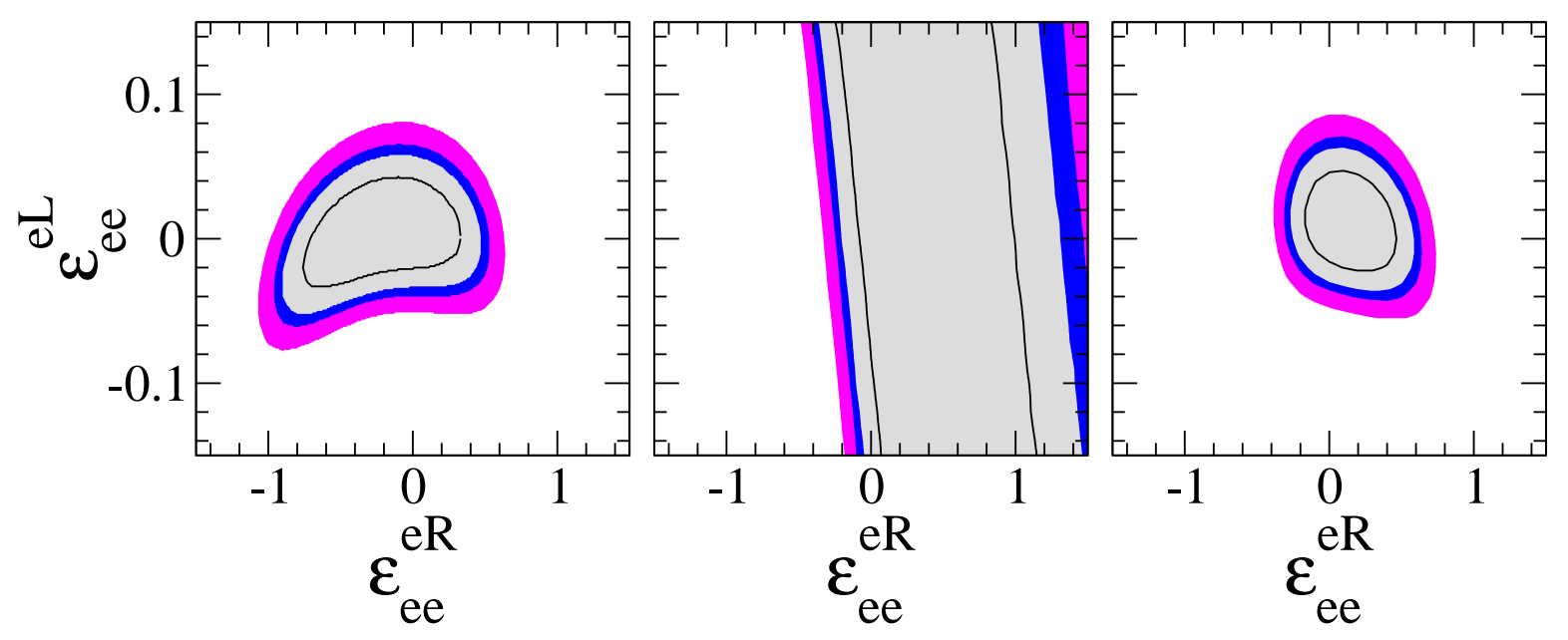

FIG. 2: Constraints on the electron neutrino non-standard interactions. Bounds at 68\%, 90\%, $95 \%$ and $99 \%$ for 2 d.o.f.. In the left panel non-standard effects are included only in the detection, in the middle panel only in propagation and in the right panel the effects are included in both processes.

rescaling of the boron flux. Second, the precision measurement of the SNO CC rate imposes a further constraint on the Super-K ES rate.

As already observed in the previous section, the constraints on the R-type coupling come from the spectral information obtained in the Super-K experiment. Current Super-K data are consistent with the spectrum predicted for standard cross-section, while still allowing for appreciable deviations. Therefore the limits on the R-type coupling are looser compared with those obtained on the L-type one (note the different scale used for $\varepsilon_{e e}^{e R}$ and $\varepsilon_{e e}^{e L}$ ). We observe that the "barycenter" of the allowed region is slightly shifted toward negative values of $\varepsilon_{e e}^{e R}(\sim-0.2)$. For such values the coefficient $g_{2}^{e} \sim 0$ and both the second and third (energy dependent) terms in Eq. (11) tend to vanish indicating a slight preference of the data for an energy independent cross section. We also observe how the allowed region is elongated towards negative values of both non-standard L-type and R-type couplings indicating that in this region of the parameter space a degeneracy exists between the second and the third term in Eq. (11). Indeed, the second term tends to give a negative tilt to the Super-K energy spectrum which is counterbalanced by the positive tilt induced by the third one (indeed its coefficient is positive in this parameter region since $g_{2}^{e}$ assumes negative values).

In the middle panel of Fig. 2 we report the constraints obtained when we include nonstandard effects only in neutrino propagation, as already discussed in Sec. II In this plane these constraints are represented by diagonal bands delimited by lines corresponding to 
constant values of the vectorial coupling. This plot clearly shows how these constraints are different and complementary to those coming from detection.

In the third panel we show the allowed region obtained by the full global analysis, where we simultaneously include non-standard effects in detection and in propagation. The effect of including NU couplings in both processes leads to an appreciable reduction of the allowed region evidencing a high complementarity and synergy of the two kinds of constraints, which effectively turns the global allowed region into a "round" shape.

It is interesting to observe that the allowed region in the third panel looks like just a naïve combination of the two regions determined separately only by detection and only by propagation. This result is important since, a priori, one would in principle expect a possible degeneracy among non-standard effects induced at the level of detection and those induced at the level of propagation. In particular, some region of the parameter space could exist where non-standard effects in detection could counterbalance those induced in the propagation process (and vice versa.) Our analysis shows, a posteriori, that such a degeneracy is instead absent. One can qualitatively understand this behavior noting that, although non-standard propagation effects could in principle partially undo the modifications induced by the non-standard detection in Super-K, their presence would unavoidably spoil the agreement of all the other experimental results (Cl, Ga, and SNO) with their respective theoretical predictions (which are all well described by standard propagation.)

We close this section quoting the range allowed [at 90\% C.L. (2 d.o.f.)] for the amplitude of the non-universal R-type coupling of electron neutrinos with electrons,

$$
-0.27<\varepsilon_{e e}^{e R}<0.59
$$

and for the L-type one,

$$
-0.036<\varepsilon_{e e}^{e L}<0.063
$$

We observe that our limits are comparable with those found by laboratory experiments [48].

\section{CONSTRAINTS ON TAU NEUTRINO INTERACTIONS}

In this section we present the numerical results of the analysis in the presence of nonuniversal couplings of $\nu_{\tau}$ with electrons. As in the case of the electron neutrinos presented 
in the previous section, also in this case we have performed a joint analysis of solar and KamLAND reactor data in the $\left(\Delta m_{21}^{2}, \sin ^{2} \theta_{12}, \varepsilon_{\tau \tau}^{e L}, \varepsilon_{\tau \tau}^{e R}\right)$ parameter space, again taking into account that only the vectorial combination of the chiral couplings enters the propagation. In contrast to the case considered in the previous section, for the $\varepsilon_{\tau \tau}^{e L}$ case the analysis is performed for a wider range than considered for $\varepsilon_{e e}^{e L}$, since the current laboratory constraints are too weak to resolve the degeneracy pattern [47].

Note that in the present case the signal observed in the Super-K experiment is the sum of the standard contribution due to scattering of the three neutrino flavors, and of an additional nonstandard contribution due to the interaction of $\tau$ neutrinos with electrons through the neutral current. These neutrinos originate from solar neutrino oscillations into a state $\nu_{a}$ which we approximate as an equal mixture of $\nu_{\mu}$ and $\nu_{\tau}$, corresponding to maximal "atmospheric" mixing angle and zero $\theta_{13}$.

Figure 3 is analogous to Fig. 2 but with the three panels showing respectively the regions allowed in the $\left[\varepsilon_{\tau \tau}^{e L}, \varepsilon_{\tau \tau}^{e R}\right]$ plane. Notice that in this case the scale of the L-type coupling is different from the case of electron neutrinos, being almost an order of magnitude larger. In the first panel, the "two-island" behavior is a manifestation of the degeneracy pattern which exists for the electron case [47] and which is not fully lifted by our current global analysis. It is clear from Eq. (111) that the neutrino electron cross section is symmetric under the simultaneous transformation $g_{1}^{a} \rightarrow-g_{1}^{a}$ and $g_{2}^{a} \rightarrow-g_{2}^{a}$. Moreover, the last term, already small due to the ratio $m_{e} / E_{\nu}$, is further suppressed compared with the electron neutrino case since its coefficient $g_{1}^{\tau} g_{2}^{\tau}$ is now smaller. Therefore, there is actually an approximate symmetry under separate changes in the sign of $g_{1,2}^{a}$. In our case this can be achieved by setting, for instance, $\varepsilon_{\tau \tau}^{e L}=-2 g_{1}^{\tau} \simeq 0.54$, which effectively amounts to the transformation $\tilde{g}_{1}^{\tau}=g_{1}^{\tau}+\varepsilon_{\tau \tau}^{e L} \rightarrow-g_{1}^{\tau}$. As can be seen in Fig. 3, our global data analysis is already able to resolve this degeneracy at $99 \%$ C.L., but is not able to resolve the same degeneracy for the $\varepsilon_{\tau \tau}^{e R}$ case. As in the case of interaction with electron neutrinos treated in the previous section we find that the "barycenter" of the allowed region is slightly shifted toward negative values of the L-type parameter, again indicating a weak preference for a energy independent differential cross section (see comments in Sec. IV).

In the middle panel, we show the constraints obtained including non standard effects only in propagation. We observe that in this case (see eq. 7) we have $\varepsilon_{\tau \tau}^{e V}=\varepsilon^{\prime} / \sin ^{2} \theta_{23} \simeq 2 \varepsilon^{\prime}$, explaining the reduced sensitivity to the vectorial coupling. Finally, the right panel is 

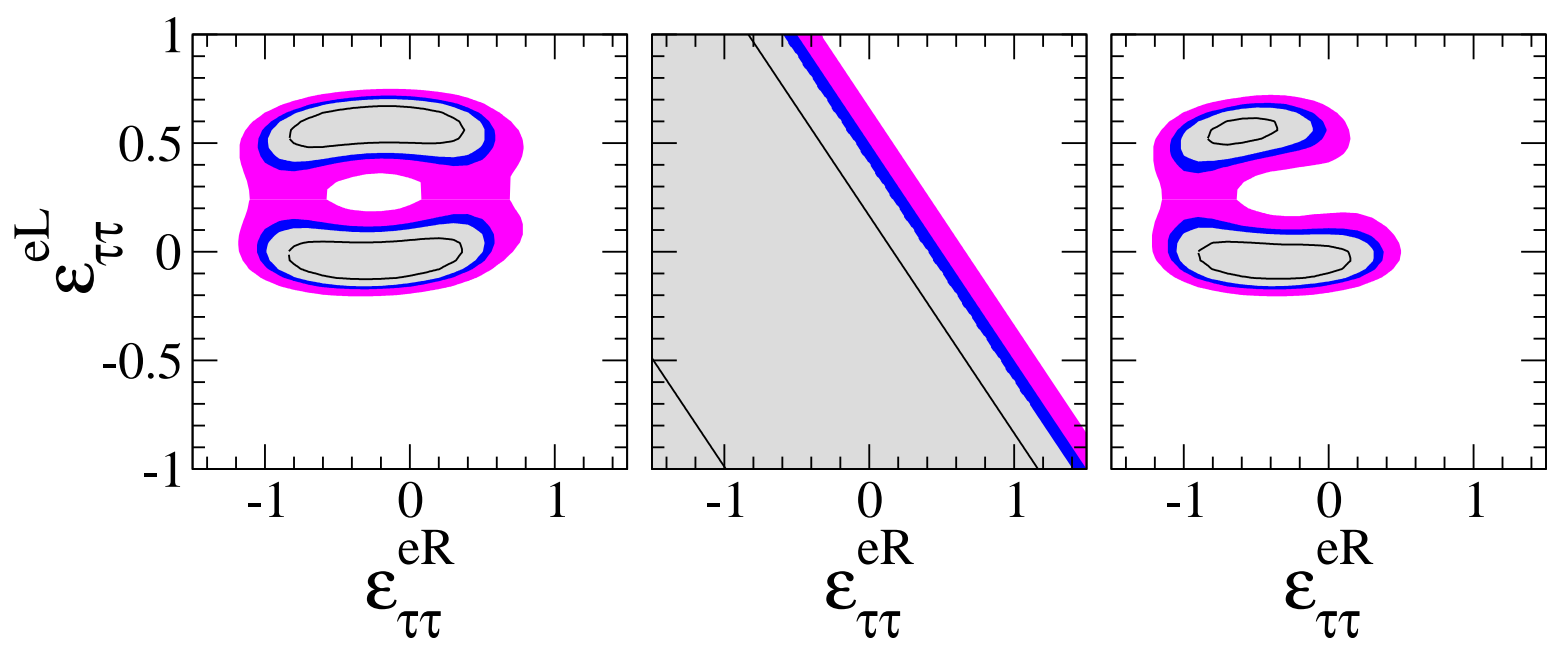

FIG. 3: Constraints on the $\tau$ neutrino non-standard interactions. Bounds at $68 \%, 90 \% 95 \%$ and 99\% for 2 d.o.f.. In the left panel non-standard effects are included only in the detection, in the middle panel only in propagation and in the right panel the effects are included in both processes. Notice the different scale for the left coupling with respect to the case of electron neutrinos presented in Fig.2.

obtained, as before, by consistently including non standard effects both in neutrino detection as well as in propagation. As for the case of electron neutrinos discussed in Sec. IV, the full analysis clearly shows the complementarity among the constraints coming from detection and propagation and the absence of any possible degeneracy between the two effects. We find the following $90 \%$ C.L. (2 d.o.f.) allowed range of the non-standard amplitude of R-type coupling:

$$
-1.05<\varepsilon_{\tau \tau}^{e R}<0.31
$$

while two disjoint ranges for the L-type coupling are obtained:

$$
-0.16<\varepsilon_{\tau \tau}^{e L}<0.11, \quad 0.41<\varepsilon_{\tau \tau}^{e L}<0.66
$$

corresponding to the "two-island" region discussed above. We observe that also in this case our limits are comparable to the existing laboratory bounds [48].

\section{SUMMARY AND CONCLUSIONS}

Motivated by neutrino mass extensions of the standard electroweak model that imply the existence of neutrino non-standard interactions, we have considered the constraints on 
the strength of effective non-universal (NU) flavor conserving four-fermion operators $\nu_{\alpha} \nu_{\alpha} \bar{e} e$ with electrons, where $\alpha=e, \tau$, that can be obtained from solar and reactor (KamLAND) neutrino data. We have consistently taken into account the effect of non-standard physics both at the level of neutrino propagation, where they modify the standard MSW behavior, as well as at the level of detection, where they affect the cross section of neutrino elastic scattering on electrons.

Our analysis allows us to trace the following important conclusions: I) The constraints on NU couplings obtained by detection and propagation of solar neutrinos are of comparable sensitivity. II) The constraints coming from the two processes are highly complementary and the general analysis allows considerable restrictions of the parameter space. III) The current data seem powerful enough to remove degeneracies possibly arising among NU couplings at the level of detection and propagation respectively. IV) The limits we find are comparable with those found by means of other model-dependent searches.

\section{Acknowledgments}

This work was supported by Spanish grants FPA2008-00319/FPA and FPA2008-01935E/FPA and ILIAS/N6 Contract RII3-CT-2004-506222. M.A.T. is supported by the DFG (Germany) under grant SFB-676. A.B. and O.G.M. were supported by Conacyt and the HELEN program.

[1] L. Wolfenstein, Phys. Rev. D17, 2369 (1978).

[2] S. P. Mikheev and A. Y. Smirnov, Sov. J. Nucl. Phys. 42, 913 (1985).

[3] B. T. Cleveland et al., Astrophys. J. 496, 505 (1998).

[4] Kamiokande collaboration, Y. Fukuda et al., Phys. Rev. Lett. 77, 1683 (1996).

[5] SAGE collaboration, J. N. Abdurashitov et al., J. Exp. Theor. Phys. 95, 181 (2002).

[6] GALLEX collaboration, W. Hampel et al., Phys. Lett. B447, 127 (1999).

[7] GNO collaboration, M. Altmann et al., Phys. Lett. B616, 174 (2005), hep-ex/0504037.

[8] R. L. Hahn, Talk at the Neutrino 2008 Conference, Christchurch, New Zealand, 2008.

[9] V. Gavrin, Talk at the Neutrino 2006 Conference, Santa Fe, New Mexico, USA, 2006.

[10] Super-Kamiokande collaboration, S. Fukuda et al., Phys. Rev. Lett. 86, 5651 (2001), hep-ex/0103032.

[11] Super-Kamiokande collaboration, S. Fukuda et al., Phys. Rev. Lett. 86, 5656 (2001), hep-ex/0103033. 
[12] Super-Kamiokande collaboration, S. Fukuda et al., Phys. Lett. B539, 179 (2002), hep-ex/0205075.

[13] Super-Kamiokande collaboration, M. B. Smy et al., Phys. Rev. D69, 011104 (2004), hep-ex/0309011.

[14] SNO collaboration, Q. R. Ahmad et al., Phys. Rev. Lett. 87, 071301 (2001), nucl-ex/0106015].

[15] SNO collaboration, Q. R. Ahmad et al., Phys. Rev. Lett. 89, 011301 (2002), nucl-ex/0204008.

[16] SNO collaboration, Q. R. Ahmad et al., Phys. Rev. Lett. 89, 011302 (2002), nucl-ex/0204009.

[17] SNO collaboration, S. N. Ahmed et al., Phys. Rev. Lett. 92, 181301 (2004), [nucl-ex/0309004].

[18] SNO collaboration, B. Aharmim et al., Phys. Rev. C72, 055502 (2005), [nucl-ex/0502021.

[19] SNO collaboration, B. Aharmim et al., Phys. Rev. Lett. 101, 111301 (2008), [0806.0989].

[20] Borexino collaboration, C. Arpesella et al., Phys. Lett. B 658, 101 (2008), [0708.2251].

[21] Borexino collaboration, C. Arpesella et al., Phys. Rev. Lett. 101, 091302 (2008), [0805.3843].

[22] Borexino collaboration, G. Bellini et al., [0808.2868].

[23] KamLAND collaboration, K. Eguchi et al., Phys. Rev. Lett. 90, 021802 (2003), hep-ex/0212021.

[24] KamLAND collaboration, T. Araki et al., Phys. Rev. Lett. 94, 081801 (2005).

[25] KamLAND collaboration, S. Abe et al., Phys. Rev. Lett. 100, 221803 (2008), [0801.4589].

[26] G. L. Fogli, E. Lisi, A. Marrone and A. Palazzo, Prog. Part. Nucl. Phys. 57, 742 (2006), hep-ph/0506083.

[27] M. Maltoni, T. Schwetz, M. A. Tortola and J. W. F. Valle, New J. Phys. 6, 122 (2004), [arXiv, hep-ph/0405172].

[28] T. Schwetz, M. Tortola and J. W. F. Valle, New J. Phys. 10, 113011 (2008), [0808.2016]; previous works by other groups as well as the relevant experimental references are given therein.

[29] G. L. Fogli et al., Phys. Rev. D 78, 033010 (2008), [0805.2517].

[30] A. Schaefer and S. E. Koonin, Phys. Lett. B 185 (1987) 417;

[31] P. I. Krastev and A. Y. Smirnov, Mod. Phys. Lett. A 6 (1991) 1001.

[32] F. N. Loreti and A. B. Balantekin, Phys. Rev. D 50, 4762 (1994) arXiv:nucl-th/9406003;

[33] H. Nunokawa, A. Rossi, V. B. Semikoz and J. W. F. Valle, Nucl. Phys. B472, 495 (1996), hep-ph/9602307].

[34] C. P. Burgess, N. S. Dzhalilov, T. I. Rashba, V. B. Semikoz and J. W. F. Valle, Mon. Not. Roy. Astron. Soc. 348, 609 (2004), astro-ph/0304462.

[35] C. Burgess et al., Astrophys. J. 588, L65 (2003), |hep-ph/0209094].

[36] C. P. Burgess et al., JCAP 0401, 007 (2004), [hep-ph/0310366].

[37] G. L. Fogli, E. Lisi, A. Marrone, D. Montanino and A. Palazzo, Phys. Rev. D 76, 033006 (2007), [0704.2568].

[38] O. G. Miranda et al., Nucl. Phys. B595, 360 (2001), hep-ph/0005259.

[39] O. G. Miranda, T. I. Rashba, A. I. Rez and J. W. F. Valle, Phys. Rev. Lett. 93, 051304 (2004), hep-ph/0311014]; O. G. Miranda, T. I. Rashba, A. I. Rez and J. W. F. Valle, Phys. Rev. D70, 113002 (2004), hep-ph/0406066.

[40] J. Schechter and J. W. F. Valle, Phys. Rev. D24, 1883 (1981), Err. D25, 283 (1982).

[41] E. K. Akhmedov, Phys. Lett. B213, 64 (1988).

[42] S. Pakvasa and J. W. F. Valle, hep-ph/0301061, Proc. of the Indian National Academy of Sciences on Neutrinos, Vol. 70A, No.1, p.189 - 222 (2004), Eds. D. Indumathi, M.V.N. Murthy and G. Rajasekaran.

[43] Z. Berezhiani, R. S. Raghavan and A. Rossi, Nucl. Phys. B638, 62 (2002), hep-ph/0111138.

[44] S. Davidson, C. Pena-Garay, N. Rius and A. Santamaria, JHEP 03, 011 (2003), 
hep-ph/0302093.

[45] A. Friedland, C. Lunardini and C. Pena-Garay, Phys. Lett. B594, 347 (2004), hep-ph/0402266.

[46] M. M. Guzzo, P. C. de Holanda and O. L. G. Peres, Phys. Lett. B591, 1 (2004), hep-ph/0403134.

[47] J. Barranco, O. G. Miranda, C. A. Moura and J. W. F. Valle, Phys. Rev. D73, 113001 (2006), hep-ph/0512195.

[48] J. Barranco, O. G. Miranda, C. A. Moura and J. W. F. Valle, Phys. Rev. D77, 093014 (2008), [0711.0698].

[49] N. Fornengo, M. Maltoni, R. Tomas Bayo, J. W. F. Valle Phys. Rev. D65, 013010 (2001), hep-ph/0108043.

[50] A. Friedland, C. Lunardini and M. Maltoni, Phys. Rev. D70, 111301(R) (2004), hep-ph/0408264.

[51] P. Huber, T. Schwetz and J. W. F. Valle, Phys. Rev. Lett. 88, 101804 (2002), hep-ph/0111224.

[52] P. Huber, T. Schwetz and J. W. F. Valle, Phys. Rev. D66, 013006 (2002), hep-ph/0202048].

[53] P. Huber and J. W. F. Valle, Phys. Lett. B523, 151 (2001), hep-ph/0108193|.

[54] N. C. Ribeiro et al., JHEP 0712, 002 (2007) [arXiv:0709.1980 [hep-ph]].

[55] J. Kopp, M. Lindner, T. Ota and J. Sato, arXiv:0710.1867 [hep-ph].

[56] J. Kopp, T. Ota and W. Winter, Phys. Rev. D78, 053007 (2008), [0804.2261].

[57] A. Esteban-Pretel, J. W. F. Valle and P. Huber, Phys. Lett. B668, 197 (2008), [0803.1790].

[58] O. G. Miranda, M. A. Tortola and J. W. F. Valle, JHEP 10, 008 (2006), hep-ph/0406280.

[59] J. Schechter and J. W. F. Valle, Phys. Rev. D22, 2227 (1980).

[60] P. Minkowski, Phys. Lett. B67, 421 (1977).

[61] M. Gell-Mann, P. Ramond and R. Slansky, (1979), Print-80-0576 (CERN).

[62] T. Yanagida, (KEK lectures, 1979), ed. Sawada and Sugamoto (KEK, 1979).

[63] R. N. Mohapatra and G. Senjanovic, Phys. Rev. Lett. 44, 912 (1980).

[64] J. Schechter and J. W. F. Valle, Phys. Rev. D25, 774 (1982).

[65] P.-H. Gu, M. Hirsch, U. Sarkar and J. W. F. Valle, 0811.0953.

[66] R. N. Mohapatra and J. W. F. Valle, Phys. Rev. D34, 1642 (1986).

[67] M. C. Gonzalez-Garcia and J. W. F. Valle, Phys. Lett. B216, 360 (1989).

[68] J. Bernabeu et al., Phys. Lett. B187, 303 (1987).

[69] G. C. Branco, M. N. Rebelo and J. W. F. Valle, Phys. Lett. B225, 385 (1989).

[70] N. Rius and J. W. F. Valle, Phys. Lett. B246, 249 (1990).

[71] J. W. F. Valle, Phys. Lett. B199, 432 (1987).

[72] H. Nunokawa, Y. Z. Qian, A. Rossi and J. W. F. Valle, Phys. Rev. D54, 4356 (1996), hep-ph/9605301.

[73] A. Esteban-Pretel, R. Tomas and J. W. F. Valle, Phys. Rev. D76, 053001 (2007), arXiv:0704.0032 [hep-ph]].

[74] L. J. Hall and M. Suzuki, Nucl. Phys. B231, 419 (1984).

[75] G. G. Ross and J. W. F. Valle, Phys. Lett. B151, 375 (1985).

[76] J. R. Ellis and et al., Phys. Lett. B150, 142 (1985).

[77] A. Santamaria and J. W. F. Valle, Phys. Lett. B195, 423 (1987).

[78] M. A. Diaz, J. C. Romao and J. W. F. Valle, Nucl. Phys. B524, 23 (1998), hep-ph/9706315.

[79] M. Hirsch, M. A. Diaz, W. Porod, J. C. Romao and J. W. F. Valle Phys. Rev. D62, 113008 (2000), hep-ph/0004115, Err-ibid. D65:119901,2002.

[80] A. Abada, S. Davidson and M. Losada, Phys. Rev. D65, 075010 (2002), hep-ph/0111332. 
[81] M. A. Diaz, M. Hirsch, W. Porod, J. C. Romao and J. W. F. Valle Phys. Rev. D68, 013009 (2003), |hep-ph/0302021].

[82] V. D. Barger, G. F. Giudice and T. Han, Phys. Rev. D40, 2987 (1989).

[83] K. S. Babu, Phys. Lett. B203, 132 (1988).

[84] A. Zee, Phys. Lett. B93, 389 (1980).

[85] M. B. Gavela, D. Hernandez, T. Ota and W. Winter, arXiv:0809.3451 [hep-ph].

[86] S. Antusch, J. P. Baumann and E. Fernandez-Martinez, Nucl. Phys. B 810, 369 (2009) arXiv:0807.1003 [hep-ph]].

[87] S. Bergmann and A. Kagan, Nucl. Phys. B538, 368 (1999), hep-ph/9803305.

[88] M. C. Gonzalez-Garcia et al., Phys. Rev. Lett. 82, 3202 (1999), |hep-ph/9809531].

[89] M. M. Guzzo, A. Masiero and S. T. Petcov, Phys. Lett. B260, 154 (1991).

[90] E. Roulet, Phys. Rev. D44, R935 (1991).

[91] Particle Data Group, C. Amsler et al., Phys. Lett. B667, 1 (2008).

[92] J. N. Bahcall, A. M. Serenelli and S. Basu, Astrophys. J. Suppl. 165, 400 (2006) arXiv:astro-ph/0511337|.

[93] A. Y. Smirnov, hep-ph/0702061.

[94] G. L. Fogli, E. Lisi, A. Marrone and A. Palazzo, Phys. Lett. B583, 149 (2004), hep-ph/0309100.

[95] J. N. Bahcall, M. Kamionkowski and A. Sirlin, Phys. Rev. D51, 6146 (1995), astro-ph/9502003|. 